\title{
An Approach for Energy-Aware Management in Ubiquitous Home Network Environment
}

\author{
Hyung-Soo Mok ${ }^{1}$, Sung-Yong Son ${ }^{2}$, Jun Hee Hong ${ }^{2}$, and Sanghoon Kim ${ }^{3}$ \\ ${ }^{1}$ The Department of Electrical Engineering, \\ Konkuk University, Gwangjin-Gu, Seoul, 143-701, Korea \\ hsmok@konkuk.ac.kr \\ ${ }^{2}$ Power IT Research Center, \\ Kyungwon University, Seongam-Si, Gyeonggi-do, 461-701, Korea \\ \{xtra, hongpa\} @kyungwon.ac.kr \\ ${ }^{3}$ The Department of Electrical and Electronics Engineering, \\ Kangwon National University, Chuncheon-Si, Gangwon-do, 200-701, Korea \\ kshoon@kangwon.ac.kr
}

\begin{abstract}
This research studies energy-aware managemen strategies in ubiquitouse home network environment. Always-on connectivity is essential to establish home networks, and it induces more energy consumption. In this work, a methodology is prosposed to achieve energy saving in overalls without harming the quality of services. Energy management technology used in wireless sensor network is expanded to information appliances, usage patterns and dependecy tree are applieds to design the management system, and the effect in energy saving is analyzed.
\end{abstract}

Keywords: Energy-Aware, Home Network, Ubiquitous, Information Appliances.

\section{Introduction}

Home network systems that have been rapidly deployed in Korea change the definition of "home" by expanding its meaning to the cyber world with the anytime and anywhere connectivity. They can check house security from outside in real time by connecting home network systems remotely with PCs, mobile phones, and telematics equipments. Even they can see and talk with the visitors who drop by during their absence. To enable these services the network needs to guarantee always-on connectivity to the internet, and every device at home must be ready to respond to the commands that may come from outside. Although these home network systems bring convenience to users, they increase energy consumption additionally. Considering world-wide tendency in energy saving due to the shortage of energy resources and the necessity of $\mathrm{CO}_{2}$ reduction, home network systems need to consider energy efficiency to be widely deployed.

Many efforts have been made to reduce energy consumption in various aspects by developing energy efficient materials and chip sets, varying CPU clocks and voltages, 
optimizing communication speed or powers, and controlling software processes, etc. Calhoun tried to reduce standby power with dynamic voltage scaling [1]. Frequency scaling is also considered in addition to voltage [2]. Dynamic power management is a common approach for embedded systems and wireless sensor networks by entering to the sleeping mode during not working $[3,4]$. The approach is especially useful when a device needs to work occasionally. Energy-aware system, routing and protocol designs are also widely performed researches [5,6,7,8,9]. Although many efforts have been conducted to reduce energy consumption for specific technology or individual device, the approach from the whole system viewpoint is insufficient.

Reducing standby power is an important and urgent issue for home appliance industry. Standby power means power dissipated while not performing the main function of a device. The power may be used only for waiting incoming commands or to accomplish side functions such as a clock and alarm. Reducing standby power under $1 \mathrm{~W}$ for home appliances is becoming a world-wide trend. However, it is difficult to define standby status in home network systems because always-on connectivity is a prerequisite for them. Since the devices should be ready to work, there is a limitation in cutting down the standby power.

It is important that energy saving in home network systems should not harm the quality of services. The quality can be divided into two view points. One is the resulting service quality induced from the activity of a home network system. For example, to control the humidity of a home environment, there are few different ways to achieve the goal. They may turn on an air conditioner, activate a dehumidifier, turn on a heater or open a window to reduce the humidity. Such an approach is not considered in this work. The other view point is whether the system can respond to a command appropriately within an expected time. To solve this problem, home network devices are classified into groups based on their allowed latency. Then, an operation strategy is proposed to reduce energy consumption.

\section{System Configurations}

Figure 1 shows an example of typical home network systems, which consists of control devices, sensor devices, information appliances, data devices and AV devices. In general, control and sensor devices use relatively low electric power because they are designed for a single purpose and do not require high computing performance. Information and $\mathrm{AV}$ devices consume much higher energy than control and sensor devices because of their complex and advanced service nature. There are many different ways in connecting these devices to a home network system, and it is difficult to determine a single standard configuration because the environment is different case by case. A network medium is selected for each device based on the functional characteristics and industry trends in this research. For sensor nodes, battery-powered Zigbee is mostly selected. Since control devices usually require relatively high electric power when it works, AC power is directly supplied to the devices when needed while using Zigbee for communication. A low speed PLC is used for information appliances, and Ethernet is used for data and AV devices. 


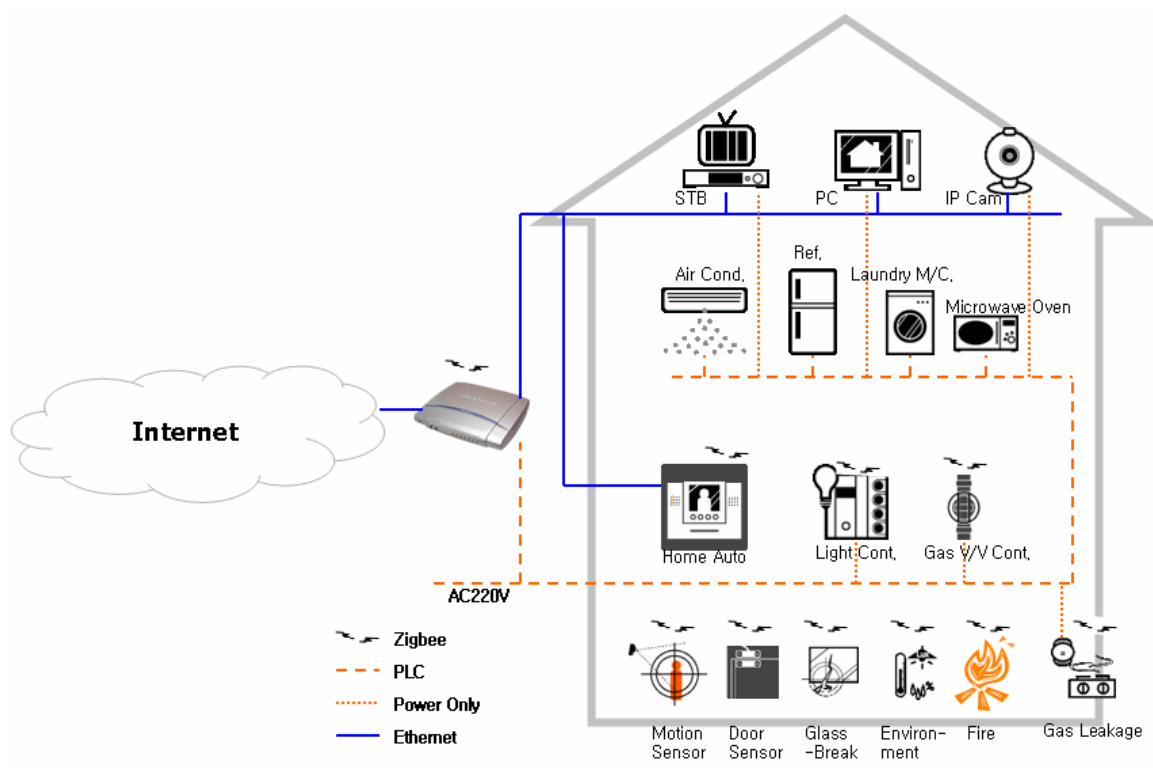

Fig. 1. A ubiquitous home network system configuration which consist of control devices, sensor devices, information appliances, data devices and AV devices

Table 1 shows the power consumption of each device in a ubiquitous home network system used in this research. Ubiquitous sensor network-based devices consume small amount of energy as well as power that ranges $25 \sim 1,500 \mathrm{~mW}$ depending on their functions. On the other hand, information appliances consume high standby power when compared to home appliances without network connectivity. The electric power of home appliances used for normal operation (air conditioning, heating, washing, etc.) is not considered in this work because they are too big ranging $300 \sim 1,500 \mathrm{~W}$ to see the effect of communications. The residential gateway and the home auto (door phone) use high standby power since they should work as communication and control hubs to accept incoming events and data.

The life of battery-powered devices has been regarded as one of the most critical issues because it is directly related to their maintenance. Therefore, efficient system design has been studied in many ways. Dynamic power management is a key approach in ubiquitous sensor networks to maximize the life of sensor nodes. To reduce power consumption, devices go to sleep mode during idle time. Much work has been done exploiting sleep state and active power management $[3,4]$.

High power consuming devices are essential to connect to the power line directly to obtain enough energy to perform their main functions. The ease of energy acquirement put the energy saving issues of those home network devices in the dead angle so far. Control devices with their own power suppliers are in the similar situation. Not enough efforts are made for those devices compared to batterypowered wireless devices. In this work, energy saving mechanism used for batterypowered wireless device is adopted. 
Table 1. The list of home network devices and their power consumption

\begin{tabular}{|c|c|c|c|c|}
\hline Device & $\begin{array}{l}\text { Number of } \\
\text { Installation }\end{array}$ & $\begin{array}{c}\text { Normal } \\
\text { (Working) }\end{array}$ & $\begin{array}{l}\text { Power Saving } \\
\text { (Waiting) }\end{array}$ & Network \\
\hline Residential Gateway & 1 & $15 W^{\prime}$ & $12 \mathrm{w}$ & Ethemet/Zigbee/PLC \\
\hline Home Auto (Door Phone) & 1 & $22 \mathrm{w}$ & $15 \mathrm{w}$ & Ethemet/Zigbee \\
\hline Motion Sensor & 3 & $<25 \mathrm{miw}$ & $<0.1 \mathrm{mi \omega i}$ & Zigbee/Battery \\
\hline Door/Window Alarm & 4 & $<90$ miwi & $<0.1 \mathrm{mi \omega i}$ & Zigbee/Battery \\
\hline Glass-Break Detector & 4 & $<100 \mathrm{miw}$ & $<0.1 \mathrm{mi \omega}$ & Zigbee/Battery \\
\hline Environment Sensor & 4 & $<100 \mathrm{miw}$ & $<0.1 \mathrm{miw}$ & Zigbee/Battery \\
\hline Fire Alarm & 3 & $<30 \mathrm{mw}$ & $<0.1 \mathrm{miv}$ & Zigbee/Battery \\
\hline Gas Detector & 1 & $1.5 \mathrm{~W}$ & $1.5 \mathrm{~W}$ & Zigbee/AC220V \\
\hline Gas Valve Controller & 1 & $3 \mathrm{wiv}$ & $1.0 \mathrm{w}$ & Zigbee/AC22OV \\
\hline Light Controller & 5 & $0.9 \mathrm{~W}$ & $0.9 \mathrm{~W}$ & Zigbee/AC220V \\
\hline Security Camera & 1 & $12 \mathrm{~W}$ & $8 W$ & Ethemet \\
\hline STB & 1 & $N / C$ & $8 W$ & Ethemet \\
\hline Computer & 1 & $\mathrm{~N} / \mathrm{C}$ & $2.3 \mathrm{w}$ & Ethemet \\
\hline Laundry & 1 & $\mathrm{~N} / \mathrm{C}$ & $8.8 \mathrm{~W}$ & PLC \\
\hline Refrigerator & 1 & $N / C$ & $10.6 \mathrm{~W}$ & PLC \\
\hline Microwave oven & 1 & $N / C$ & $5.7 \mathrm{~W}$ & PLC \\
\hline Air Conditioner & 1 & $\mathrm{~N} / \mathrm{C}$ & $3.8 \mathrm{~W}$ & PLC \\
\hline
\end{tabular}

\section{System Analysis and Results}

The functions of devices used in home networks are classified into 4 basic types depending on the nature of data that they generate or use. Event sources generate urgent signals that should affect to other home network systems immediately. Event sinks accept data generated from event sources, so they should be always ready to get data transferred from other nodes. Information sources generate data that may be important but not urgent, and information sinks accept data generated from information sources. The types can be represented as the latency sensitiveness in their services. Although devices developed to perform a singe function are easy to determine their types, some devices are difficult to be classified into a category because they may perform complex functions. Table 2 shows an example of types and corresponding devices.

Table 2. An example of device type classfications for home network devices

\begin{tabular}{|c|c|c|}
\hline Type & Devices & $\begin{array}{c}\text { Latency } \\
\text { Sensitiveness }\end{array}$ \\
\hline Event Source & $\begin{array}{c}\text { Motion Sensor, Door/Window Alarm, Glass-Break Detector, } \\
\text { Fire Alarm, Gas Detector }\end{array}$ & Low \\
\hline Data Source & Environment Sensor & High \\
\hline Event Sink & Gas Valve Controller, Light Controller & Low \\
\hline Data Sink & Laundry, Refrigerator, Microwave oven, Air Conditioner & High \\
\hline Data Hub & Residential Gateway, Home Auto & \\
\hline
\end{tabular}


In real life, the device types can vary depending on the status of home, which is determined from given situations. The status can be represented with life modes such as Stay, Going-out, and Sleeping. In each mode, the types of devices can be differently defined. For example, a motion detector is important in Sleeping and Going-out modes to monitor unexpected intrusions. However, it may generate false alarm by the interference from the movements of family members in Stay mode. Therefore, the detector would be better to be used to collect and analyze life patterns in the mode. Although a light control needs to respond to commands immediately in Stay and Sleeping mode, slow response would not make a serious problem in Goingout mode. For that reason, the types of devices should be determined for each mode to set up appropriate power management strategies. Table 3 shows an example of devices and their types for given life modes.

Table 3. The types of devices corresponding to life modes

\begin{tabular}{|c|c|c|c|}
\hline Device & Stay & Sleeping & Going-out \\
\hline Residential Gateway & Esource, Esink, Dsource, Dsink & ESource, Esink, Dsource, Dsink & ESource, Esink, Dsource, Dsink \\
\hline Home Auto (Door Phone) & Esource, Esink, Dsource & Esource, Esink, Dsource & Esource, Esink, Dsource \\
\hline Motion Sensor & $\mathrm{N} / U$ & Esource & Esource \\
\hline Door/Window Alarm & $\mathrm{N} / U$ & Esource & Esource \\
\hline Glass-Break Detector & $\mathrm{N} / U$ & Esource & Esource \\
\hline Environment Sensor & Dsource & Dsource & Esource \\
\hline Fire Alarm & Esource & Esource & Esource \\
\hline Gas Detector & Esource & Esource & Dsink \\
\hline Gas Valve Controller & Esink & Dsink & Dsink \\
\hline Light Control & Esink & Esink & Dsink \\
\hline Security Camera & N/U & N/U & Dsink \\
\hline STB & Dsource & Dsink & Dsink \\
\hline Computer & Dsource & Dsink & Dsink \\
\hline Laundry & Esink & Dsink & Dsink \\
\hline Refrigerator & Esink & Dsink & Dsink \\
\hline Microwave oven & Esink & Dsink & \\
\hline Air Conditioner & Esink & & \\
\hline
\end{tabular}

Battery-powered sensor nodes are energy efficient enough compared to other devices in this case. They generally consume less than $100 \mathrm{~mW}$ and $0.1 \mathrm{~mW}$ for working modes and power save modes, respectively, as shown in Table 1. When considering the standby powers of information appliances and IP devices that range $2 \sim 10 \mathrm{~W}$, the electric power used by sensor nodes are negligible. This does not mean that there is no room to be improved in energy management for sensor networks, but more efforts are required for other devices to reduce energy consumption. The home network system used in this work as it is consumes 2,019Wh per day. The electric energy is mostly used by IP devices and information appliances, which amounts 1,109Wh and 693.6Wh, respectively. Although IP devices spend 54\% of entire energy, it is difficult to reduce the power consumption of IP devices because they serve as an event and data sink of the system providing network connectivity to the 
entire system. To receive all events and data generated by other devices without missing, some nodes should be awake all the time. Residential gateways and home auto devices usually do the work in home networks. Though other IP devices such as computers, STBs, and security cameras generally provide complex services, their roles are limited in home networks. Reducing energy consumptions of IP devices is an important issue because they use more than $50 \%$ of entire energy.

Information appliances are found to consume more energy than expected when comparing with home appliances which recently tend to be designed to use less than $1 \mathrm{~W}$ for standby power. By using efficient network communication modules, it is possible to reduce the energy consumption for information appliances. In this example, the energy consumption becomes to $1,747 \mathrm{Wh}$ per day, and it is $13 \%$ in overall, and $30 \%$ without IP devices less than the original amount.

Then, sleeping mechanism is added to remove unnecessary energy waste. Except for some emergency cases, devices can provide the equivalent services without losing the service quality with small amount of response delay. Not to miss incoming events or commands, the devices can contact a service hub right after waking up, and check up incoming signals. Since latency is allowed to a certain degree except for the event sink devices, sleeping can be an effective tool in energy saving as proved in batterypowered wireless devices.

To analyze the energy consumption of the home network system, 3 life patterns are designed as shown in Fig 2. In pattern A, all family members stay outside during daytime. Pattern B and C represent families who spend more time at home than ones with pattern $\mathrm{A}$.

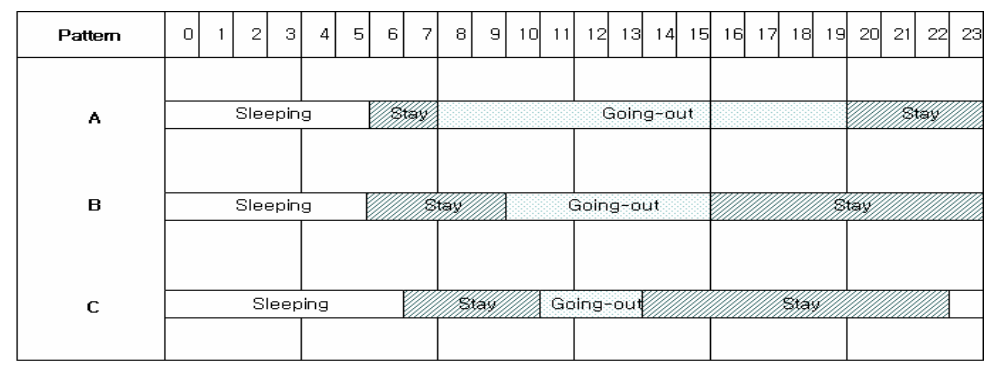

Fig. 2. Three life patterns are designed to analyze the power consumption of a home network system

Figure 3 shows the result of energy used in the home network system after applying two strategies for life pattern A. For the first step, efficient communication modules are applied to information appliances and control devices. This mainly decreased the energy used by information appliances. In the second step, the sleeping mechanism is applied and the energy consumption is decreased $31 \%$ of the original amount using 1,390Wh. Without considering IP devices, $70 \%$ of the original amount is diminished. The energy consumptions for life pattern $\mathrm{B}$ and $\mathrm{C}$ are obtained as $1,426 \mathrm{Wh}$ and $1,438 \mathrm{Wh}$, respectively. 


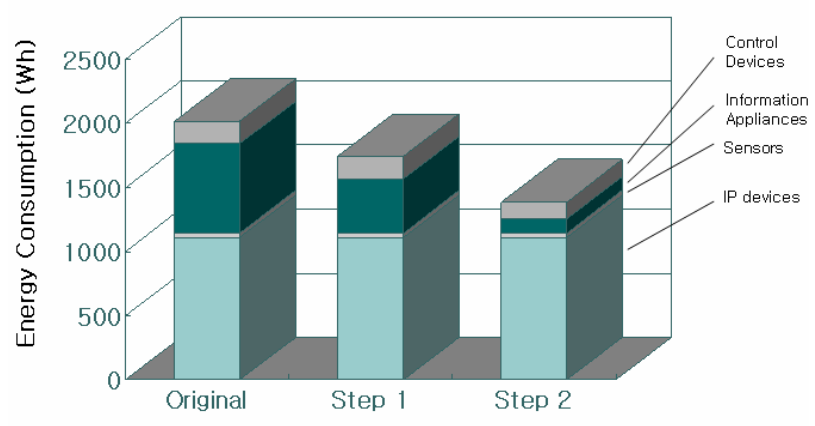

Fig. 3. The energy consumption per day of the selected home network system is shown. In step 1 , network communication nodes are improved to be power efficient, and sleeping mechanism is added in step 2 for a life pattern $A$ in Figure 2. Most reduction occurs in control devices and information appliances in this work.

Dependency trees among devices in a home network system are useful to make energy management policies. For example, VCR, STB, and DVD are usually dependent on $\mathrm{TV}$, and monitors, printers and scanners are hardly used without computers. By using the relations, a hierarchical energy management can be designed.

\section{Conclusions}

In this work, a home network system is modeled and analyzed to figure out energy consumption characteristics. Since external electric power is provided to most devices in home networks, no much work has been done to save energy so far. Especially, IP devices and information appliances have much to be improved. Energy efficient hardware and sleeping mechanisms which are used for battery-powered wireless devices are expanded to other home network devices. Device types are classified for each life mode to reduce unnecessary communications, and the possibility of energy saving is shown.

\section{References}

1. Calhoun, B.H, Chandrakasan, A.P.: Standby Power Reduction Using Dynamic Voltage Scaling and Canary Flip-Flop Structures. IEEE Journal of Solid-State Circuits 39(9), 1504 1511 (2004)

2. IBM and MotaVista Software: Dynamic Power Management for Embedded System. Ver.1.1, IBM and MotaVista (November 19, 2002)

3. Brock, B., Rajamani, K.: Dynamic Power Managent for Embedded Systems. In: IEEE International System-On-Chip (SOC) Conference, pp. 416-419. IEEE Computer Society Press, Los Alamitos (2003)

4. Sinha, A., Chandrakasan, A.: Dynamic Power Management in Wireless Sensor Networks. IEEE Design and Test of Computers 19(2), 62-74 (2001) 
5. Calhoun, B.H., et al.: Design Considerations for Ultra-low Energy Wirelss Microsensor Nodes. IEEE Transactions on Computers (June 2005)

6. Sohrabi, K., Gao, J., Ailawadhi, V., Pottie, G.J.: Protocols for Self-Organization of a Wireless Sensor Network. IEEE Personal Communications 7(5), 16-27 (2000)

7. Raghunathan, V., Schurgers, C., Park, S., Srivastava, M.B.: Energy-Aware Wireless Microsensor Networks. IEEE Personal Communications 7(2), 40-50 (2002)

8. Schurgers, C., Raghunathan, V., Srivastava, M.: Power Management for Energy-aware Communication System. ACM Transactions on Embedded Computing Systems 2(3), 431447 (2004)

9. Heo, J., Yi, S., Park, G., Cho, Y., Hong, J.: EAR-RT: Energy Aware Routing with RealTime Guarantee for Wireless Sensor Networks. In: Alexandrov, V.N., van Albada, G.D., Sloot, P.M.A., Dongarra, J.J. (eds.) ICCS 2006. LNCS, vol. 3991, pp. 946-953. Springer, Heidelberg (2006) 